\title{
A report of amelanotic malignant melanoma of the esophagus diagnosed appropriately with novel markers: A case report
}

\author{
JUNYA KOBAYASHI $^{1}$, DAISUKE FUJIMOTO ${ }^{2}$, MAKOTO MURAKAMI $^{1}$, YASUO HIRONO ${ }^{1}$ and TAKANORI GOI $^{1}$ \\ ${ }^{1}$ Department of Surgery 1, Faculty of Medicine, University of Fukui, Fukui 910-1193; ${ }^{2}$ Department of Surgery, \\ Teikyo University Hospital, Kawasaki, Kanagawa 213-8507, Japan
}

Received January 13, 2018; Accepted April 5, 2018

DOI: $10.3892 /$ ol.2018.8479

\begin{abstract}
The present case study reported of amelanotic malignant melanoma of the esophagus. A 68-year-old man underwent laparoscopic distal gastrectomy for early gastric cancer diagnosis. After gastrectomy, endoscopic examination revealed a protruded lesion lying adjacent to the melanosis area of the esophagus. Histology of the biopsy specimen suggested malignancy, but the diagnosis could not be made. The patient underwent trans-thoraco-abdominal curative subtotal esophagectomy. Immunohistochemical examination of the resected specimen was negative for HBM-45 and Melan-A. However, immunohistochemical examinations of SOX10 (Sry-related HMg-Box gene 10) and KBA.62, which are not associated with melanosome, were strongly positive, and tyrosinase was notably positive. A diagnosis primary of amelanotic malignant melanoma of the esophagus that consisted of only premelanosomes was made. The present findings suggest that, in the diagnosis of malignant melanoma, SOX10 and KBA.62 may be useful, particularly in diagnosing amelanotic malignant melanoma.
\end{abstract}

\section{Introduction}

Primary malignant melanoma of the esophagus (PMME) is rare, accounting for $0.1-0.2 \%$ of all primary malignancie of the esophagus (1). Moreover, amelanotic malignant melanoma accounts for $10-25 \%$ of all malignant melanomas of the esophagus and is a rarer tumor with a rapid progression and a poor prognosis, often progressing with multiple metastases even in the early stage of the disease; only a few case reports have been published in the literature (1). It is difficult to diagnose PMME, especially the amelanotic type, with the surgically

Correspondence to: Dr Daisuke Fujimoto, Department of Surgery, Teikyo University Hospital, Mizonokuchi, Mizonokuchi 3-8-3, Takatsu-ku, Kawasaki, Kanagawa 213-8507, Japan

E-mail: rui5218@gmail.com

Key words: amelanotic malignant melanoma, SOX10, KBA.62, melanosome resected specimen or endoscopic biopsy tissue. Pigment cells of normal and malignant melanocytes are useful for the analysis of observation differentiation (2). True amelanotic malignant melanomas produce no melanin or granules, resulting in no pigmentation and contain stage I and/or II melanosomes (3). Accordingly, primary amelanotic malignant melanoma of the esophagus is frequently misdiagnosed at biopsy as poorly differentiated squamous cell carcinoma, sarcoma, spindle cell carcinoma, or undifferentiated carcinoma. We report a case of primary amelanotic malignant melanoma of the esophagus that was difficult to diagnose but could be radical resection, and the review of the literature regarding the usefulness of new markers in diagnosis.

\section{Case report}

A 68-year-old man underwent laparoscopic curative distal gastrectomy for early gastric cancer two years ago. Pathological diagnosis had been stage IA: T1bNOM0 according to the TNM classification of the International Union Against Cancer. He had smoked 20 cigarettes per day at the age of 20 to 36 years and drunken $720 \mathrm{ml}$ of Japanese rice wine every day till the age of 48 years. His physical examination showed the scars of laparoscopic distal gastrectomy. He also underwent endoscopic examination every year. One year after the gastrectomy, endoscopic examination revealed the formation of melanosis in the middle thoracic esophagus (Fig. 1A). One year later, endoscopic examination revealed the expansion of the melanosis area and the appearance of a protruded lesion lying adjacent to the melanosis area (Fig. 1B). It was a type 0 -Is non-pigmented tumor with a central recess, $20 \mathrm{~mm}$ in longitudinal diameter, with a clear round wall. Lugol staining method of endoscopic examination gave a negative result. Magnifying endoscopy demonstrated a vascular area over $3 \mathrm{~mm}$ and that the vascular is extreme distention: the finding of type B3, that was the magnifying endoscopic classification of the Japan Esophageal Society (4) (Fig. 1C). Endoscopic ultrasonography demonstrated that the tumor was communicated with the second layer. The third layer disappeared by the invasion of the tumor (Fig. 1D). These findings indicated that the depth of the tumor was beyond muscularis propria. Histology of the biopsy specimen showed anisocytosis, nuclear enlargement, high N/C ratio, prominent nucleoli and vacuoles. Immunohistochemical staining was positive for $\mathrm{S}-100$, but negative for cytokeratin 

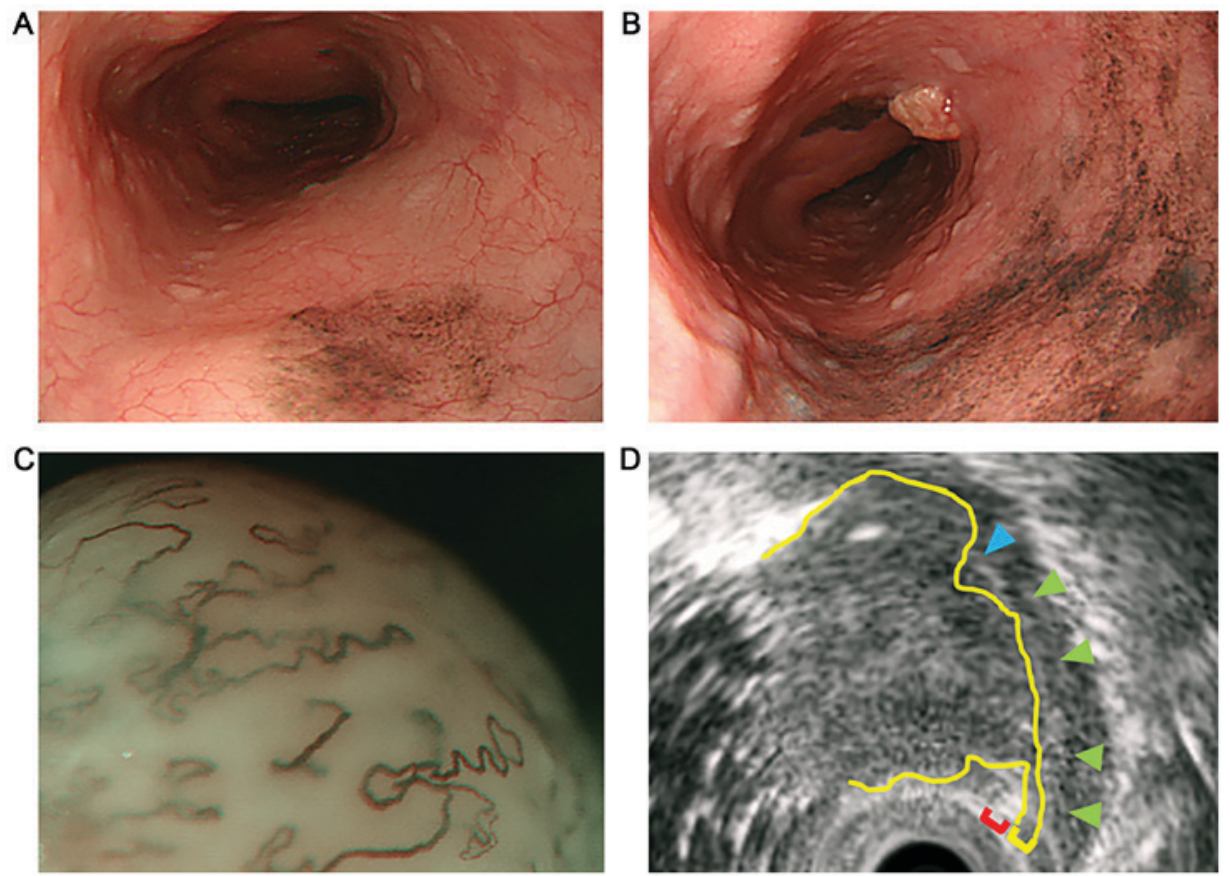

Figure 1. (A) First time after gastrectomy, upper gastrointestinal scope showed only melanosis. (B) Two years after gastrectomy, upper gastrointestinal scope showed an elevated tumor and surrounding melanosis. (C) Magnifying endoscopy demonstrated the vascular structure, which exhibited extreme distention. (D) Endoscopic ultrasonography indicated the standard five-layer image of esophagus wall. The hyperechoic layer indicated with the red parenthesis is the first layer (interface between fluid in the lumen and the superficial mucosa). The next hypoechoic layer, indicated by the yellow parenthesis, revealed the second layer (lamina propria and muscularis mucosa) and communicated with the tumor surrounded by yellow line. The hyperechoic layer, indicated by green arrows, is the third layer (submucosa and interface between submucosa and muscularis propria), and this layer disappeared at the blue arrow. This finding suggests that tumor invaded the third layer.
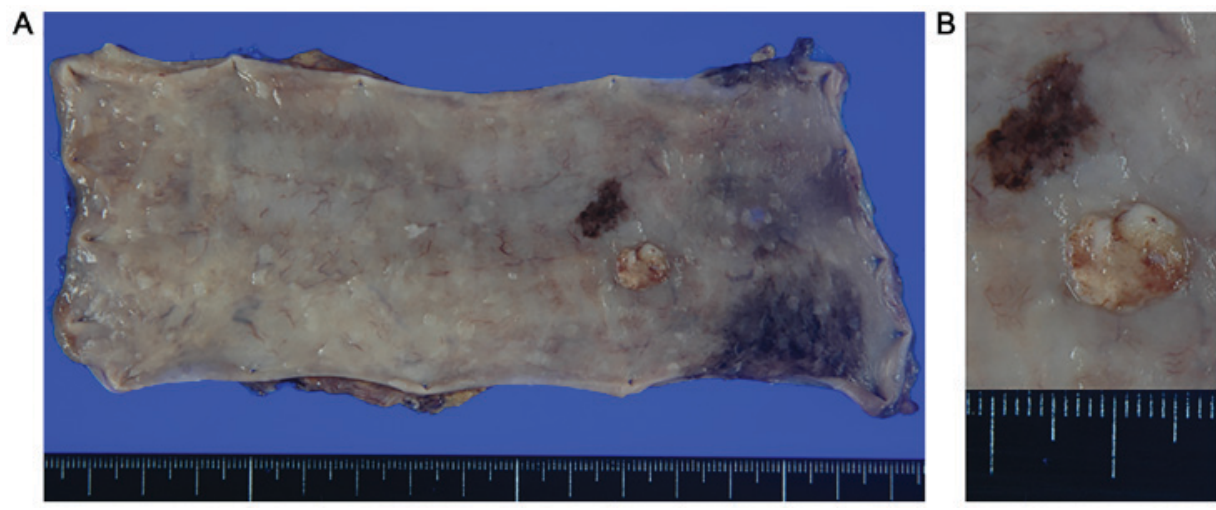

Figure 2. Macroscopic appearance. (A and B) A non-pigmented granular lesion was indicated.

AE1/AE3, desmin, $\alpha$-SMA, CD34, Leukocyte common antigen, HMB-45, Melan-A, c-kit and DOG-1. We could make a diagnosis of malignant tumor but could not reach a definite histological type. Enhanced computed tomography from chest to pelvis did not demonstrate the primary mass and metastases. There was accumulation in the middle of the esophagus and no accumulations of lymph nodes and other organs in positron-emission tomography. We did not have the accurate diagnosis, but we confirmed the malignancy and the necessity of the surgery. We decided to resect it. The patient underwent trans-thoraco-abdominal curative subtotal esophagectomy. Reconstruction was performed by pulling up the colon via the retrosternal route; the site of anastomosis was in the neck. The surgical specimen demonstrated a 20x15 mm non-pigmented granular protruded lesion with a central recess next to melanosis (Fig. 2A and B). It was located in only submucous coat and did not invade the muscularis mucosae (Fig. 3A). The tumor consisted of a circular small atypical cell with anisocytosis, nuclear enlargement and prominent nucleoli (Fig. 3B). There was the junctional change, the identified findings of malignant melanoma (Fig. 4A and B). Immunohistochemical staining was positive for $\mathrm{S}-100$ (Fig. 5A) and negative for HBM-45 and Melan-A (Fig. 5B and C), and partially positive for tyrosinase (Fig. 5D). These results did not reveal the diagnosis. Since S-100 was positive by immunohistochemical staining, several differential diagnoses (such as rhabdomyo sarcoma, synovial sarcoma, malignant peripheral nerve sheath tumor, and undifferentiated cancer) were candidates (5). We added the immunohistochemical examination of SOX10 (Sry-related HMg-Box gene 10) and KBA.62. It was positive 
A
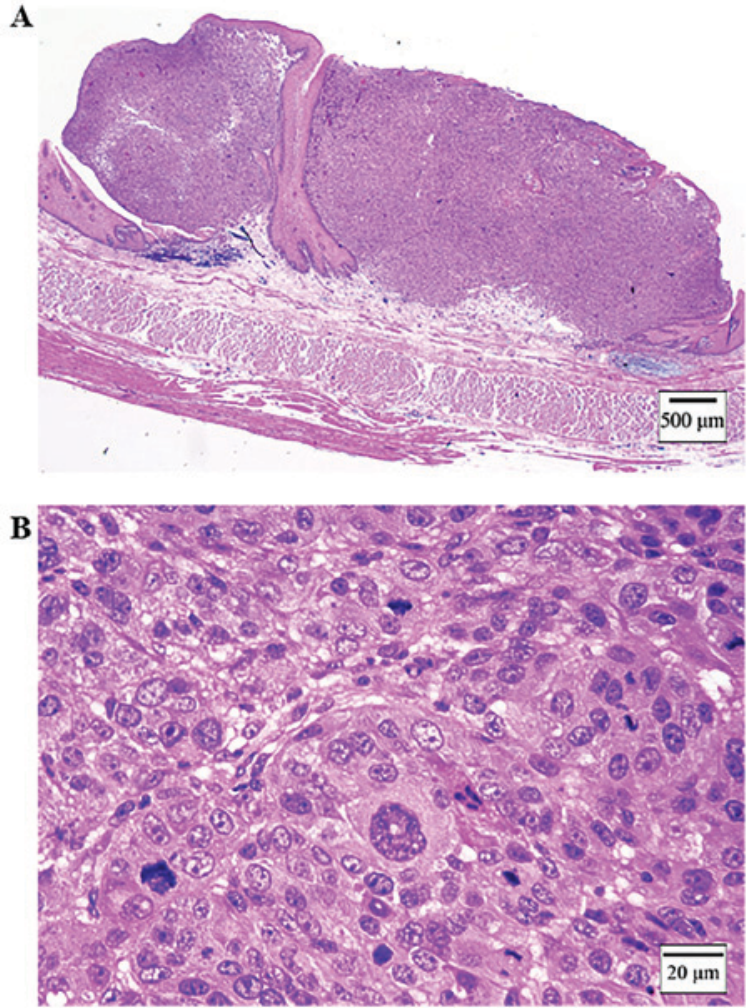

Figure 3. Hematoxylin and eosin staining. (A) Hematoxylin and eosin staining indicated the tumor did not invade the muscularis propria (magnification, x12.5). (B) Hematoxylin and eosin stain indicated a circular small atypical cell with anisocytosis (magnification, $\mathrm{x} 400$ ).
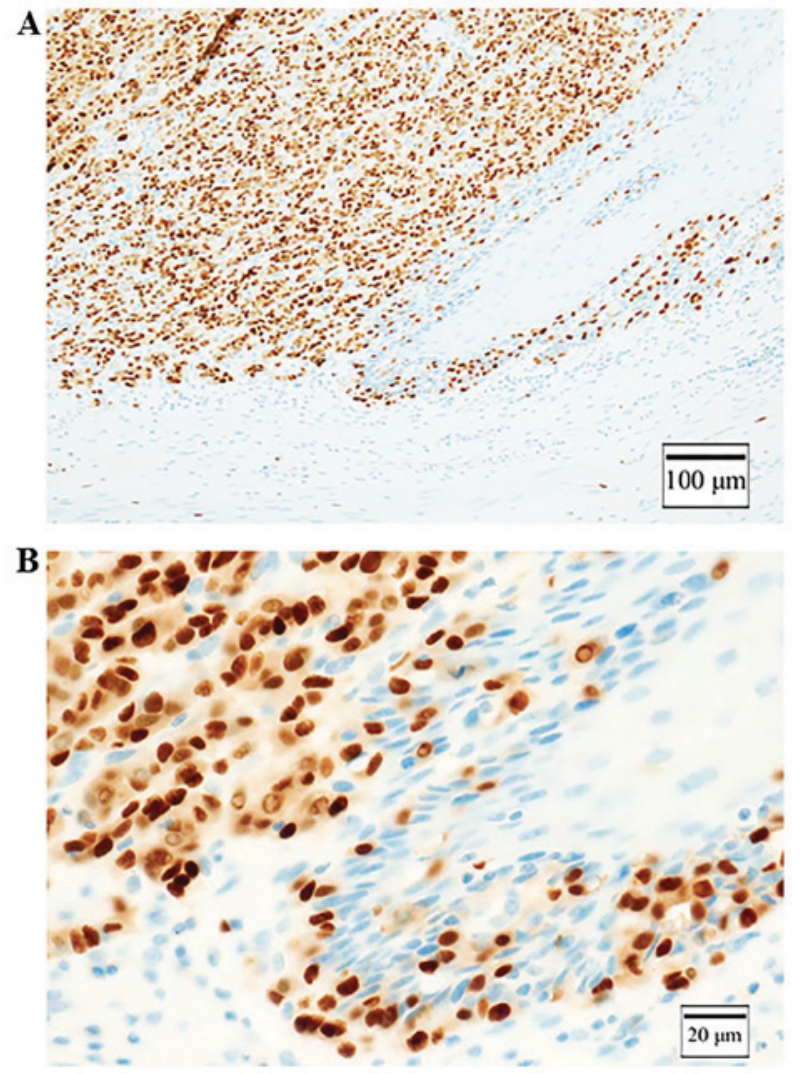

Figure 4. SOX10 immunohistochemical stain. (A and B) In the esophageal epithelial mucosa that was contacted with the SOX10 positive tumor mass, SOX10 positive tumor cells infiltrated from the basal side of the esophagus, indicating junctional changes. Magnification (A), x100; (B), x400. for SOX10 and KBA.62 (Fig. 5E and F). We could make a diagnosis amelanotic malignant melanoma with effort, because we confirmed the junctional change which is a histological feature of malignant melanoma, and furthermore, in the immunohistochemical staining test, SOX10, KBA.62, S-100, and tyrosinase were positive in tumor cells. There are no recurrence findings 1.5 year after the surgery.

\section{Discussion}

Amelanotic malignant melanoma of the esophagus is a rare disease, and only 15 such cases have been accumulated from 1996 to 2017 (6-15). Only one cases stated long survival (6). Other case reports stated a mean survival of approximately 9 months (7-15). Amelanotic malignant melanoma produces no melanin pigments, and accounts for approximately $2 \%$ of all malignant melanomas of the esophagus (10). PMME occurs mainly in the sixth and seventh decades of life, but may develop at any age, with a male-to- female ratio of 2:1 (10). Volpon recommended surgical resection as the treatment for PMME as it results in a longer mean survival than chemo- or radiotherapy alone (14 vs. 3 months) (16). It is also difficult to diagnose primary amelanotic malignant melanoma because melanin pigment is absent. Diagnosis criteria of PMME are i) a typical histological pattern of melanoma and the presence of melanin granules within the tumor cells; ii) an origin in an area of junctional change within the squamous epithelium; and iii) junctional change with melanotic cells in the adjacent epithelium $(1,17)$. The junctional change means melanocytic proliferation in the junctional zone between the dermis and the epidermis with its derivatives (18). Allen and Spitz reported that the presence of junctional activity is the most importance factor for diagnosis (17). In this case, we found the tumor at an early stage, and the structure of junctional change remained. But it was very hard to reach any definite diagnosis. This is because the markers, which were conventionally used in the diagnosis of malignant melanoma, were not useful and the endoscopic biopsy tissues were very small for confirming the structure of junctional change.

S-100, HMB-45 and Melan-A are conventionally useful marker to discriminate melanomas from other tumors. But in this case, the conventional maker of immunohistochemical staining was positive only S-100 and negative for the others. SOX10 and KBA.62 are relatively new markers of malignant melanoma and both were positive in this case. As an immunochemical feature of SOX10 and KBA.62, Sox10 was consistently expressed in benign Schwann cell tumors of soft tissue and the GI-tract and metastatic melanoma, and was variably present in malignant peripheral nerve sheath tumors, in contrast, Sox 10 was absent in many potential mimics of nerve sheath tumors such as cellular neurothekeoma, meningioma, gastrointestinal stromal tumors, PEComa, and a variety of fibroblastic-myofibroblastic tumors (19). KBA.62 recognized an unknown determinant expressed in melanoma cells and commonly maliganant melanoma. This antibody was found immunoreactivity in most metastatic melanomas, desmoplastic melanomas, and well-differentiated squamous carcinomas (20). The sensitivity and specificity of S-100 were reported to be $97-100$ and $75-87 \%$, respectively. The sensitivity of HMB-45 was $69-93 \%$. The sensitivity and 

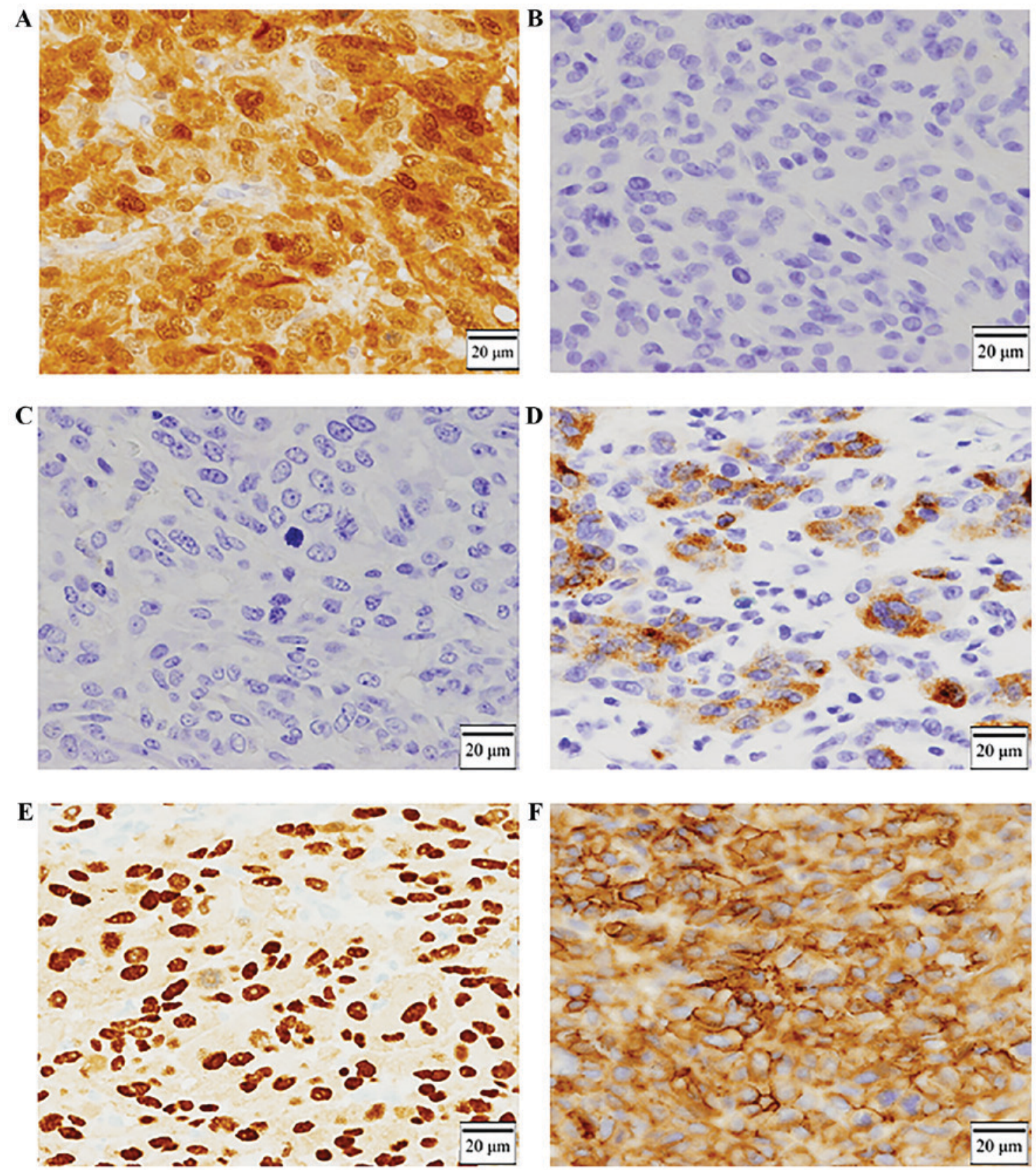

Figure 5. (A) S-100 immunohistochemical staining was strongly positive. (B) HBM-45 immunohistochemical staining was negative. (C) Melan-A immunohistochemical staining was negative. (D) Tyrosinase immunohistochemical staining was partially positive. (E) SOX10 immunohistochemical staining was strongly positive. (F) KBA.62 immunohistochemical staining was also strongly positive. Magnification, x400.

specificity were 75-92 and 95-100\% for Melan-A, and 84-94 and $97-100 \%$ for tyrosinase, respectively (3). The sensitivity of SOX10 was reported to be $97-100 \%$, whereas the sensitivity of KBA62 was $93 \%$ (3). SOX10 and KBA.62 are not relative to melanosome. SOX10 is a transcriptional activator of microphthalmia-associated transcription factor (MITF), MITF regulates the differentiation and development of melanocytes and retinal pigment epithelium and is also responsible for pigment cell-specific transcription of the melanogenesis enzyme genes (21). The SOX10-MITF pathway was involved in maintaining the proliferative and tumorigenic ability, cell cycle regulation, expression of survival factors, and metastasis formation in melanoma cells (22). It is also considered to be important for the specification, maturation, and maintenance of melanocytes (23). Furthermore, it has been shown to be a sensitive and specific marker for spindle cell and desmoplastic melanomas (23). KBA.62 was detected in 1995 as a new monoclonal antibody against a melanoma-associated antigen and reacted with all histopathologic subtypes of nevi, including junctional, intradermal, compound, Spitz, and dysplastic (24). In malignant melanoma, but it is unknown which determinant expressed KBA62 recognizes, and the function of KBA62 as a protein is also unknown (25). The sensitivity of anti-S-100 and KBA.62 antibodies in detecting occult melanoma metastasis was similar, moreover, KBA.62 identified melanoma patients who had confirmed sentinel lymph node metastasis but were negative for HMB-45 (25).

Melanosomes exist in four distinct stages as they become increasingly laden with melanin pigment prior to their transportation out of the cell into neighboring keratinocytes 
via melanocyte dendrites. Stage I and II melanosomes are known as early melanosomes because they have not initiated melanin synthesis. Amelanotic malignant melanoma consists of melanosome stage I and/or II, this is the reason for no pigment of amelanotic malignant melanoma (3). Both markers, HMB-45 and Melan-A, has been associated with stage II melanosome (26). Although we did not observe it under electron microscopy, the reason for negative HMB-45 and Melan-A may be that, our case of amelanotic malignant melanoma consisted of only melanosome stage I (also called premelanosomes). None of the melanosome stage II showed that immunohistochemical staining was negative for HMB-45 and Melan-A. Tyrosinase, partially positive in our case, is necessary for the synthesis of melanin (27). Tyrosinase is a key enzyme in melanin synthesis that can catalyze three different reactions: The hydroxylation of tyrosine to 3,4-dihydroxyphenylalanine (DOPA), the oxidation of DOPA to DOPA quinone and the oxidation of 5,6-dihydroxyindole to indole-quinone (28). In melanomas, tyrosinase can be seen as fine granular cytoplasmic staining (29). Positive staining tends to be strong and diffusive (30). The sensitivity of tyrosinase for melanoma is somewhat better than HMB45, and sensitivity decreases with increasing clinical stage and in metastatic lesions (30). The specificity of tyrosinase for melanoma is $97-100 \%$ (31). Tyrosinase has been found in rare angiolipomas, a minority of angiomyolipomas and clear cell sarcomas of the tendon sheath, and pigmented nerve sheath tumors (30). Melanin synthesis occurs within the melanosome, a specific lysosome-related organelle that matures through four morphologic stages (I-IV), and stage I melanosomes are spherical vacuoles that lack tyrosinase activity and melanin (32-34). Some amelanotic melanoma cells contain significant levels of catalytically inactive tyrosinase molecules and the levels of pigmentation in mammalian melanocytes are regulated by a tyrosinase activation process (35). We think that in our case, tyrosinase was partially positive, but not active. S-100, SOX10 and KBA.62 were not related to melanosome and the expression of melanin, such that immunohistochemical staining was positive for them. In fact, Cecile reported that immunohistochemical staining was negative for HMB-45 and positive for KBA.62 in the case of amelanotic malignant melanoma (25). Tissue staining with conventional makers including HBM-45, Melan-A and S-100, and histological features are useful for leading to the diagnosis of PMME. However, when the sample size is small like an endoscopic biopsy tissue, histological features are often not recognizable. In our case, it may be impossible to diagnose with conventional markers. In such cases, we believe that SOX10 and KBA.62 can be useful new markers in the diagnosis of PMME, especially in amelanotic malignant melanoma of the esophagus. If it is possible to investigate the melanogenesis ability of the tumor with as electron microscope, the diagnosis will becomes easier. In our research, it was the limitation that we did not examine the tumor with electron microscope.

In conclusion, HMB-45 and Melan-A, the generally used diagnostic markers of malignant melanoma, were negative in our case. Based on our findings, SOX10 and KBA.62 can be considered as the new markers for the diagnosis of amelanotic malignant melanosome.

\section{Acknowledgements}

The authors would like to thank Dr. Yoshiaki Imamura (Pathological Department, University of Fukui, Fukui, Japan) for his helpful comments.

\section{Funding}

No funding was received.

\section{Availability of data and materials}

The datasets used and/or analyzed during the current study are available from the corresponding author on reasonable request.

\section{Author's contributions}

DF, JK, MM, YH and TG were part of the upper gastrointestinal surgical treatment team that treated the present case. DF was the attending doctor of the patient and was involved in the followed up the patient after surgery. JK, MM and TG contributed to the preoperative diagnosis, and JK, YH and DF operated on the patient. The final diagnosis of the patient was made by all authors. JK and DF provided major contributions in writing manuscript. $\mathrm{YH}$ and MM provided major contributions in immunohistochemical staining. TG revised the manuscript critically for important intellectual content. All authors read and approved the final manuscript.

\section{Ethics approval and consent to participate}

This participant signed an informed consent agreement.

\section{Consent for publication}

The patient provided consent for publication of the data.

\section{Competing interests}

The authors declare that they have no competing interests.

\section{References}

1. Stringa O, Valdez R, Beguerie JR, Abbruzzese M, Lioni M, Nadales A, Iudica F, Venditti J and San Roman A: Primary amelanotic melanoma of the esophagus. Int J Dermatol 45: 1207-1210, 2006.

2. Slominski A, Tobin DJ, Shibahara S and Wortsman J: Melanin pigmentation in mammalian skin and its hormonal regulation. Physiol Rev 84: 1155-1228, 2004.

3. Naoki $\mathrm{O}$ and Akira K: The stage of melanogenesis in amelanotic melanoma. In: Melanoma in the clinic-diagnosis, management and complications of malignancy. Mandi M (ed). InTech, London, pp277-286, 2011.

4. Japan esophageal society: Japanese classification of esophageal cancer, 11th edition: Part I. Esopahgus 14: 1-36, 2017.

5. Ordóñez NG: Value of melanocytic-associated immunohistochemical markers in the diagnosis of malignant melanoma: A review and update. Hum Pathol 45: 191-205, 2014.

6. Hirayama Y, Masahiro T, Tanaka T, Ishihara M, Ohnishi S, Hara K, Mizuno N, Hijioka S, Okuno N, Abe T, et al: Slow-growing amelanotic malignant melanoma of the esophagus with long survival: A case report and review of the literature. Endosc Int Open 5: E1076-E1080, 2017.

7. Ramaswamy B, Bhandarkar AM, Venkitachalam S and Trivedi S: Amelanotic malignant melanoma of the cervical oesophagus. BMJ Case Rep 2014: pii: bcr2014204182, 2014 
8. Terada T: Amelanotic malignant melanoma of the esophagus: Report of two cases with immunohistochemical and molecular genetic study of KIT and PDGFRA. World J Gastroenterol 15 : 2679-2683, 2009.

9. Wang S, Thamboo TP, Nga ME, Zin T, Cheng A and Tan KB: C-kit positive amelanotic melanoma of the oesophagus: A potential diagnostic pitfall. Pathology 40: 527-530, 2008.

10. Kranzfelder M, Seidl S, Dobritz M and Brücher BL: Amelanotic esophageal malignant melanoma: Case report and short review of the literature. Case Rep Gastroenterol 2: 224-231, 2008.

11. Stringa O, Valdez R, Beguerie JR, Abbruzzese M, Lioni M, Nadales A, Iudica F, Venditti J and San Roman A: Primary amelanotic melanoma of the esophagus. Int J Dermatol 45: 1207-1210, 2006.

12. De Simone P, Gelin M and El Nakadi I: Amelanotic malignant melanoma of the esophagus. Report of a case. Minerva Chir 61 45-49, 2006.

13. Suzuki Y, Aoyama N, Minamide J, Takata K and Ogata T: Amelanotic malignant melanoma of the esophagus: Report of a patient with recurrence successfully treated with chemoendocrine therapy. Int J Clin Oncol 10: 204-207, 2005.

14. Heidemann J, Lebiedz P, Herbst H, Spahn TW, Domagk D, Domschke W and Kucharzik T: Amelanotic malignant melanoma of the esophagus: Case report. Z Gastroenterol 43: 597-600,2005

15. Lee SH, Park SH, Kim HG and Kim CB: Primary malignan melanoma of the esophagus. Yonsei Med J 39: 468-473, 1998.

16. Volpin E, Sauvanet A, Couvelard A and Belghiti J: Primary malignant melanoma of the esophagus: A case report and review of the literature. Dis Esophagus 15: 244-249, 2002.

17. Allen AC and Spitz S: Malignant melanoma; a clinicopathological analysis of criteria for diagnosis and prognosis. Cancer 6 : $1-45,1953$.

18. Levene A: On the histological diagnosis and prognosis of malignant melanoma. J Clin Pathol 33: 101-124, 1980

19. Miettinen M, McCue PA, Sarlomo-Rikala M, Biernat W, Czapiewski P, Kopczynski J, Thompson LD, Lasota J, Wang Z and Fetsch JF: Sox10-a marker for not only schwannian and melanocytic neoplasms but also myoepithelial cell tumors of soft tissue: A systematic analysis of 5134 tumors. Am J Surg Pathol 39: 826-835, 2015.

20. Kaufmann O, Koch S, Burghardt J, Audring H and Dietel M: Tyrosinase, melan-A, and KBA62 as markers for the immunohistochemical identification of metastatic amelanotic melanomas on paraffin sections. Mod Pathol 11: 740-746, 1998.

21. Shibahara S, Takeda K, Yasumoto K, Udono T, Watanabe K, Saito $\mathrm{H}$ and Takahashi K: Microphthalmia-associated transcription factor (MITF): Multiplicity in structure, function, and regulation. J Investig Dermatol Symp Proc 6: 99-104, 2001.

22. Tudrej KB, Czepielewska E and Kozłowska-Wojciechowska M: SOX10-MITF pathway activity in melanoma cells. Arch Med Sci 13: 1493-1503, 2017.

23. Nonaka D, Chiriboga L and Rubin BP: Sox10: A pan-schwannian and melanocytic marker. Am J Surg Pathol 32: 1291-1298, 2008.
24. Cohen-Kanfo E, al Saati T, Aziza J, Ralfkiaer E, Selves J, Gorgiet B and Delsol G: Production and characterisation of an antimelanoma monoclonal antibody KBA.62 using a new melanoma cell line reactive on paraffin wax embedded sections. J Clin Pathol 48: 826-831, 1995.

25. Pagès $C$, Rochaix $P$, al Saati $T$, Valmary-Degano $S$, Boulinguez $S$, Launay F, Carle P, Lauwers F, Payoux P, Le Guellec S, et al: KBA.62: A useful marker for primary and metastatic melanomas. Hum Pathol 39: 1136-1142, 2008.

26. Chen KG, Leapman RD, Zhang G, Lai B, Valencia JC, Cardarelli CO, Vieira WD, Hearing VJ and Gotttesman MM: Influence of melanosome dynamics on melanoma drug sensitivity. J Natl Cancer Inst 101: 1259-1271, 2009.

27. Tief K, Hahne M, Schmidt A and Beermann F: Tyrosinase, the key enzyme in melanin synthesis, is expressed in murine brain. Eur J Biochem 241: 12-16, 1996.

28. Hearing VJ and Tsukamoto K: Enzymatic control of pigmentation in mammals. FASEB J 5: 2902-2909, 1991.

29. Hofbauer GF, Kamarashev J, Geertsen R, Böni R and Dummer R: Tyrosinase immunoreactivity in formalin-fixed, paraffinembedded primary and metastatic melanoma: Frequency and distribution. J Cutan Pathol 25: 204-209, 1998

30. Orchard GE: Comparison of immunohistochemical labelling of melanocyte differentiation antibodies melan-A, tyrosinase and HMB 45 with NKIC3 and S100 protein in the evaluation of benign naevi and malignant melanoma. Histochem J 32: 475-481, 2000 .

31. Jungbluth AA, Iversen K, Coplan K, Kolb D, Stockert E, Chen YT, Old LJ and Busam K: T311-an anti-tyrosinase monoclonal antibody for the detection of melanocytic lesions in paraffin embedded tissues. Pathol Res Pract 196: 235-242, 2000.

32. Paterson EK, Fielder TJ, MacGregor GR, Ito S, Wakamatsu K, Gillen DL, Eby V, Boissy RE and Ganesan AK: Tyrosinase depletion prevents the maturation of melanosomes in the mouse hair follicle. PLoS One 10: e0143702, 2015.

33. Słominski A, Moellmann G, Kuklinska E, Bomirski A and Pawelek J: Positive regulation of melanin pigmentation by two key substrates of the melanogenic pathway, L-tyrosine and L-dopa. J Cell Sci 89: 287-296, 1988.

34. Slominski A, Moellmann G and Kuklinska E: L-tyrosine, L-dopa, and tyrosinase as positive regulators of the subcellular apparatus of melanogenesis in Bomirski Ab amelanotic melanoma cells. Pigment Cell Res 2: 109-116, 1989.

35. Fuller BB, Iman DS and Lunsford JB: Comparison of tyrosinase levels in amelanotic and melanotic melanoma cell cultures by a competitive enzyme-linked immunoadsorbent assay and by immunotitration analysis. J Cell Physiol 134: 149-154, 1988.

This work is licensed under a Creative Commons Attribution-NonCommercial-NoDerivatives 4.0 International (CC BY-NC-ND 4.0) License. 